\title{
Novel Clinically Weight-Optimized Dynamic Conformal Arcs (WO-DCA) for Liver SBRT: A Comparison with Volumetric Modulated Arc Therapy (VMAT)
}

\author{
Yucel Saglam ${ }^{1,2}$ \\ Yasemin Bolukbasi ${ }^{1-3}$ \\ Ali Ihsan Atasoy' \\ Fatih Karakose ${ }^{1}$ \\ Mustafa Budak' \\ Vildan Alpan ${ }^{1,2}$ \\ Erkan Topkan (iD) \\ Ugur Selek (iD ${ }^{1-3}$
}

'Koc University, School of Medicine, Department of Radiation Oncology, Istanbul, Turkey; ${ }^{2}$ UT MD Anderson Radiation Oncology Outreach Center at American Hospital, Istanbul, Turkey; ${ }^{3}$ University of Texas, MD Anderson Cancer Center, Department of Radiation Oncology, Houston, TX, USA; ${ }^{4}$ Baskent University Medical Faculty, Department of Radiation Oncology, Adana, Turkey
Purpose: To evaluate the feasibility of shortening the duration of liver stereotactic radiotherapy (SBRT) without jeopardizing dosimetry or conformity by utilizing weight-optimized dynamic conformal arcs (WO-DCA) as opposed to volumetric modulated arc therapy (VMAT) for tumors away from critical structures.

Methods: Nineteen patients with liver metastasis were included, previously treated with 50 Gy in 4 fractions with VMAT technique using two partial coplanar arcs of $6 \mathrm{MV}$ beams delivered in high-definition multi-leaf collimator (HD-MLC). Two coplanar partial WODCA were generated on Pinnacle treatment planning system (TPS) for each patient; and MLC aperture around the planning target volume (PTV) was automatically generated at different margins for both arcs and maintained dynamically around the target during arc rotation. Weight of the two arcs using optimization method was adjusted between the arcs to maximize tumor coverage and protect organs at risk (OAR) based on the RTOG-0438 protocol.

Results: The WO-DCA plans successfully "agreed" with the standard VMAT for OAR (liver, spinal cord, stomach, duodenum, small bowel, and heart) and PTV ( $D_{\text {mean }}, D_{98 \%}, D_{2 \%}$, $\mathrm{CI}$, and GI), with superior mean quality assurance (QA) pass rate (97.06 vs 93.00 for VMAT; $\mathrm{P}<0.001$ and $\mathrm{t}=8.87$ ). Similarly, the WO-DCA technique additionally reduced the beam-on time (3.26 vs 4.43; $\mathrm{P}<0.001)$ and monitor unit (1860 vs 2705 for VMAT; $\mathrm{P}<0.001)$ values significantly.

Conclusion: The WO-DCA plans might minimize small-field dosimetry errors and defeat patient-specific VMAT QA requirements due to the omission of MLC beam modulation through the target volume. The WO-DCA plans may additionally enable faster treatment delivery times and lower OAR without sacrificing target doses in SBRT of liver tumors away from critical structures.

Keywords: liver, stereotactic body radiotherapy, dynamic conformal arcs, weight optimization, VMAT

\section{Introduction}

The liver is among the most frequent sites for metastatic spread for many tumors, particularly colorectal cancers. ${ }^{1}$ Patients with a limited number of liver metastases (LM) are viewed as excellent candidates for local therapy, for whom surgery is still the best treatment choice for long-term local control of oligometastatic disease. ${ }^{2,3}$
Koc University, School of Medicine Department of Radiation Oncology,

Davutpasa Caddesi, No: 4, 340I0,

Topkapi, Istanbul, Turkey

Email ugurselek@yahoo.com 
Although $10-20 \%$ of these patients are fit for surgery, the rest needs alternative nonsurgical local therapies, including stereotactic body radiotherapy (SBRT), radiofrequency ablation (RFA), microwave ablation, cryoablation, transarterial chemoembolization, and alcohol injection. ${ }^{4,5}$ SBRT, as a noninvasive modality, has gained recognition in the last two decades, with Phase I and II studies demonstrating efficacy in delivering large doses of radiation to the target volume while sparing healthy liver tissue and the organs at risk (OAR) with extraordinary sensitivity and low toxicity profile, ${ }^{6-10}$ specifically with a mild acceptable risk of radiation-induced liver disease (RILD). ${ }^{11-13}$

Liver SBRT plans principally rely upon the practically identical principles of intracranial stereotactic radiotherapy, mostly delivered in 1-5 fractions (up to 8-10 fractions), adopting a cumulative biologically effective dose goal of $\geq 100$ Gy. ${ }^{14-16}$ Motion management embodies a significant challenge in abdominal SBRT like its thoracic counterpart, which might get further complicated in larger target volumes. ${ }^{17-19}$ The customary ways to deal with motion have entailed the expansion of the gross or clinical target volume to include the entire range of motion after Wolthaus et al's portrayal of 5 strategies for motion management; ${ }^{10}$ defined as the internal target volume (ITV). However, in any case, there is a lack of consensus for the SBRT treatment planning or delivery technique despite the implementation of a wide range of software and hardware systems in the last decade. ${ }^{14}$

Volumetric modulated arc therapy (VMAT), a rotational intensity-modulated radiation therapy (IMRT), has become a well-recognized treatment technique with an ITV approach in liver SBRT using the synchronous unique dynamic movement of the multi-leaf collimator (MLC) leaves. Nevertheless, highly modulated IMRT/VMAT plans are sensitive to delivery uncertainties due to the background interaction of multiple beams of MLC modulation and possible small-field dosimetry errors and interplay effects dependent on the abdominal tumor and liver motion during treatment. Current optimization strategies do not restrict leaf movement to prevent the target from clogging and are prone to possible dosimetric errors termed the "interplay effect" because of unforeseen interactions between the organ motion and MLC leaf motion. This deleterious effect might promote dosimetric deviations beyond $20 \%$, which could be averaged out over traditionally fractionated $(>25)$ courses of IMRT.

While lung SBRT studies have attested the necessity of using multiple arcs to obtain the average benefit for hypo- fractionated VMAT (Dynamic conformal arcs for lung stereotactic body radiation therapy: A comparison with volumetric-modulated arc therapy), an alternative technique such as dynamic conformal arcs (DCA) has been proposed to reduce or eliminate concerns of the interplay effect brought about by MLC movement in small areas. ${ }^{20-}$

${ }^{25}$ In this respect, Pokhrel et al uncovered that hybrid 3Ddynamic conformal arc (h-DCA) therapy, a blend of the dynamic conformal arcs and static fields, in lung radiotherapy minimized the small-field dosimetry and MLC interplay effects with ensuant improved target coverage. ${ }^{26}$ In the same way, Ross et al modified the dynamic conformal arc (MDCA) technique for lung SBRT and documented improved quality and PTV coverage with shortened treatment times in several clinical models. $^{22}$

On this core background, we have extrapolated this information from lung radiotherapy and investigated the strategy of weight-optimized dynamic conformal arcs (WO-DCA) in liver SBRT for metastatic tumors at least $2 \mathrm{~cm}$ away from critical structures, in accordance with the rationale of more reduced geometric complexity, and therefore, reduced risk for interplay errors prompted by respiratory motion.

\section{Materials and Methods Patients' Selection}

The institutional review board approved the design of the present dosimetric study before the acquisition of any information. Our dosimetric comparison research protocol comprised 19 patients meeting the inclusion criteria of a homogeneous cohort with the baseline characteristics displayed in Table 1: presented with single liver metastases; tumors at least $2 \mathrm{~cm}$ away in all directions of any critical structures including the ribs, diaphragm, duodenum, stomach, colon, major portal vessels, common and main bile ducts, and heart; planned with the same treatment technique and the number of arcs (double partial arc VMAT, $182^{\circ}-0^{\circ}$ and $\left.0^{\circ}-182^{\circ}\right)$ in the same version treatment planning system (TPS); prescribed the same dose of $50 \mathrm{~Gy}$ in 4 fractions (12.5 Gy/fraction), treated in the same MLC system (HD MLC, Varian TrueBeam, Varian Medical Systems, Palo Alto, CA) between 2017 to December 2019. The exclusion criteria included primary liver tumors, metastases located at or involving the portalhilar structures, previous liver SBRT history, synchronous $\geq 2$ liver metastases. 
Table I Patients' Characteristics

\begin{tabular}{|c|c|c|c|c|c|}
\hline Patient No & Age & Tumor Localization & PTV (cc) & ITV (cc) & Healthy Liver Volume (cc) \\
\hline I & 61 & Segment VIII & 76.7I & 39.28 & $|79| .5 \mid$ \\
\hline 2 & 49 & Segment VIII & 8.00 & 2.89 & 1776.78 \\
\hline 3 & 52 & Segment VII & 19.05 & 10.79 & 1502.40 \\
\hline 4 & 54 & Segment IV & 44.96 & 26.38 & 1362.07 \\
\hline 5 & 74 & Segment II & 79.22 & 52.09 & I202.1I \\
\hline 6 & 41 & Segment VIII & 6.856 & 2.50 & 1236.89 \\
\hline 7 & 79 & Segment V & 18.64 & 4.93 & | 4997.27 \\
\hline 8 & 48 & Segment IV & 8.63 & 3.53 & 1086.28 \\
\hline 9 & 48 & Segment IV & 12.04 & 5.40 & 1403.26 \\
\hline 10 & 58 & Segment IV & 18.54 & 8.17 & 1602.90 \\
\hline II & 69 & Segment VIII & 3.71 & 2.51 & 1074.27 \\
\hline 12 & 61 & Segment II & 25.50 & 12.24 & 958.50 \\
\hline 13 & 71 & Segment VII & 30.21 & 14.16 & 1728.13 \\
\hline 14 & 52 & Segment II & 8.83 & 2.71 & || $30.4 \mid$ \\
\hline 15 & 52 & Segment VIII & 23.92 & 11.06 & 1167.12 \\
\hline 16 & 64 & Segment II & 32.40 & 18.20 & 1282.49 \\
\hline 17 & 66 & Segment V-VIII intersection & 8.05 & 2.42 & 1121.87 \\
\hline 18 & 61 & Segment V-VIII intersection & 95.63 & 60.56 & 1104.22 \\
\hline 19 & 49 & Segment IV & 61.47 & 39.67 & 1594.99 \\
\hline
\end{tabular}

Abbreviations: cc, cubic centimeter-volume; PTV, planning target volume; ITV, internal target volume; Healthy Liver Volume, extracted liver volume from ITV; Segment, the Couinaud classification of liver anatomy divided into eight functionally independent segments.

\section{Imaging, Patient Immobilization, and Target Definition}

All patients were immobilized using the Body Pro-Lok TM platform (CIVCO system, Orange City, IA, USA) in the supine position with their arms above their head using an A-bar and knee-foot stopper immobilization (CIVCO, Kalona, Iowa). The free-breathing contrast-enhanced simulation CT scan was acquired on Philips Brilliance Big Bore 16 slice CT scanner (Philips Medical Systems Inc., Cleveland, $\mathrm{OH}$ ) at 1-mm slice thickness. The isocenter was determined by the radiation oncologist at the probable center of the metastatic tumor to be marked with 3 green laser systems (DORADO 3 of LAP Laser, Lüneburg, Germany) for alignment reference. Following the free-breathing scan, all patients underwent a respiration-correlated 4D-CT scan using the Varian RPM system (version 1.7.5) in the same position. The 4D-CT images were reconstructed in 10 equally spaced phase bins using a Tumor-LOC 4D workstation on virtual simulations software (Philips Medical Systems Inc., Cleveland, $\mathrm{OH}$ ), where the maximum intensity projection (MIP) images were generated. The regular 3D CT and the MIP images were imported into Philips Pinnacle Treatment Planning System 9.10 (Philips Medical Systems Inc., Cleveland, $\mathrm{OH}$ ) and co-registered for target delineation. The simulation procedure was performed using the imaging conditions specified previously for each patient. Individual treatment plans utilizing the VMAT techniques to a total dose of 50 Gy administered in 4 daily fractions were generated for each patient. All gross target volumes (GTV) were delineated by an experienced senior radiation oncologist (US), while GTV was modified covering tumor in all 10 respiratory phases of motion in addition to reference MIP images, finalized as internal GTV equaling to the ITV. Our institutional standard PTV for VMAT SBRT contained automated $0.3 \mathrm{~mm}$ circumferential expansion of the ITV surfaces to create PTV, accounting for patient setup error per RTOG $-0438 .{ }^{27}$ All plans were normalized to at least $95 \%$ of the volume of PTV to be covered by the 50 Gy isodose line. The primary goal during planning and comparison was defined as similar PTV coverage for all approaches as defined previously; while, the secondary goal constituted that the mean dose to the liver (healthy liver organ = Liver-GTV) should be $<16 \mathrm{~Gy}$, dose for $50 \%$ of the liver is less than $15 \mathrm{~Gy}\left(\mathrm{D}_{50 \%}<15 \mathrm{~Gy}\right)$ and a minimum of $700 \mathrm{cc}$ liver tissue should be spared from $15 \mathrm{~Gy}\left(\mathrm{~V}_{\text {Liver }}\right.$ $\left.-\mathrm{V}_{15 \mathrm{~Gy}} \geq 700 \mathrm{cc}\right)$, the maximum dose to spinal cord $<18$ Gy, stomach $<28$ Gy, duodenum $<28$ Gy, small bowel $<$ $28 \mathrm{~Gy}$, and heart $\mathrm{V}_{40 \mathrm{~Gy}}<10 \%$ to avoid side effects. 


\section{Clinical VMAT Planning}

The VMAT plans were carefully designed for each patient on the Philips Pinnacle Treatment Planning System 9.10 (Philips Medical Systems Inc., Cleveland, $\mathrm{OH}$ ) via a collapsed cone convolution (CCC) algorithm, according to our standard approach of double partial arcs having the same isocenter rotating clockwise and counter-clockwise starting from $182^{\circ}$ and $0^{\circ}$. Multiple control points having 356 segments in double partial arcs were created using the smart arc optimization (Smart Arc) algorithm in Pinnacle. The control point described gantry speed, dose rate, total delivery time, and leaf travel speed. All plans were delivered on Varian TrueBeam (Varian Medical Systems, Palo Alto, CA) with a maximum dose rate of $600 \mathrm{MU} / \mathrm{min}$. A grid size of $0.3 \times 0.3 \times 0.3 \mathrm{~cm} 3$ was employed for all required calculations. All plans were generated by an experienced senior medical radiation physicist (YS) and were departmentally peer-reviewed according to the institutional guidelines. Comparison of a selected MLC control point (one control point for arc 1 on each plan) between the WO-DCA and VMAT plans with $182^{\circ}-0^{\circ}$ beam's-eyeview (BEV) on a digitally reconstructed radiograph (DRR) of the same patient was demonstrated in Figure 1.

\section{WO-DCA Planning}

The standard clinical VMAT plans were retrospectively replanned for each patient (in Pinnacle 9.10) using a novel feature (weighting optimization) with DCA-based dose calculation followed by VMAT optimization. The WODCA (with identical beam geometry) plans rely upon utilizing two partial arcs with the same isocenter rotating clockwise and counter-clockwise starting from $182^{\circ}$ and $0^{\circ}$. See Figure 2 for the proposed workflow of this novel approach.

For the first arc (clockwise), a $2 \mathrm{~mm}$ MLC margin around the PTV was dynamically created and maintained around the target during arc rotation. For the second arc (counter-clockwise), MLC margins of $0 \mathrm{~mm}$ around the PTV in the lateral, anterior, and posterior directions, and of $1 \mathrm{~mm}$ in the cranial-caudal directions were generated. The second arc with a different margin in every direction allowed a change in the isodose distributions in the target volume and tapered the heterogeneity in dose distribution within the target volume laterally on PTV occurring due to the half-arcs and the body contour. The MLCs of DCA arcs were monitored to dynamically conform to the BEV projections of the PTV for each arc in Pinnacle 9.10. Besides, the MLC diaphragm shape was accustomed to a very high priority in the controller and continued to calculate a DCA-based 3D dose distribution. Before launching the weight optimization, we initiated the plan with a $50 \%$ weight for two half arcs. Next was weight optimization using VMAT optimization with identical planning objectives, dose calculation algorithm, grid size, and convergence mode identical to the original clinical VMAT plan, including the normal tissue objective parameters and ring structures.

The WO-DCA optimization used to determine the weights of the DCA arcs was carried out with the same constraints for OARs and the same normalization volume to achieve the same coverage used in the VMAT technique
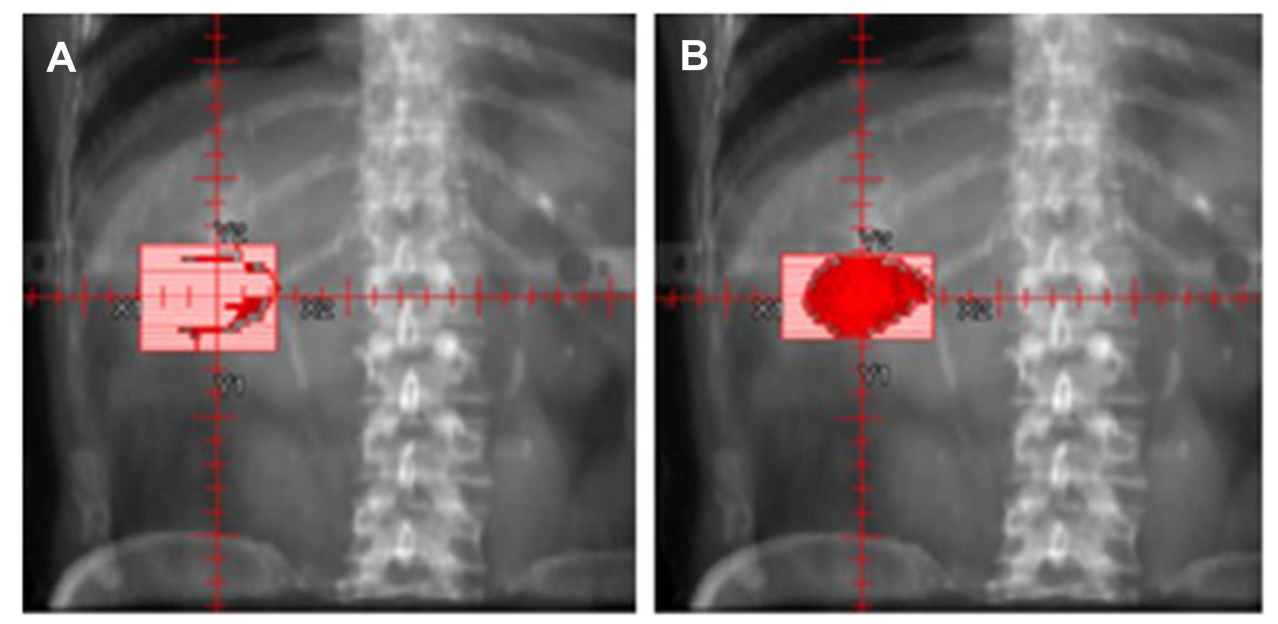

Figure I Beam eye view of multi-leaf collimator control point (one control point for arc \#I on each plan): (A) VMAT beam modulated by multi-leaf collimator masking red color PTV and (B) WO-DCA beam on an example patient $\left(182^{\circ}-0^{\circ}\right)$ on DRR displaying that the red color PTV volume is visible due to avoidance of PTV blocking by the multi-leaf collimators. 


\section{Create DCA arcs}

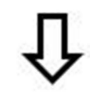

\section{Fit the MLC aperture with checking the PTV}

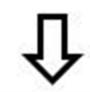

\section{Calculate the basic DCA plan}

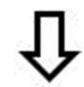

\section{Optimize the weight on DCA plan with the same VMAT constraints}

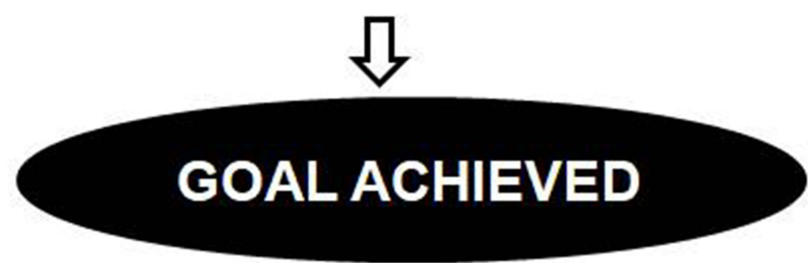

Figure 2 The study algorithm of WO-DCA optimization.

in the original Pinnacle plan (a feature in Pinnacle v9.10, beam weight algorithm in calculation models). The final dose calculation was performed with the CCC algorithm in Pinnacle TPS whenever the target was achieved. As shown in Figure 1, the WO-DCA MLC pattern (right panel) conformed to the PTV (red) while the majority of the PTV was under the MLC block, due to MLC modulation, in the clinical VMAT plan (left panel).

\section{Patient-Specific Quality Assurance (QA) and Treatment Delivery}

As per our institutional guidelines, all QA procedures including patient-specific QA, a daily QA check on kilovoltage to megavoltage, and imaging isocenter coincidences were completed before the delivery of the SBRT. $^{28}$ For each plan having VMAT and WO-DCA, a corresponding patient-specific QA plan was also created for measurement in OmniPro I'mRT software version 1.4.3.3 (Scanditronix Wellhofer AB Sweden) with Kodak EDR-2 Ready pack film (Eastman Kodak Company,
Rochester, NY) on VIDAR's Dosimetry PRO $^{\circledR}$ Advantage film scanner (VIDAR Systems Corporation, Herndon, VA). The film scanner was calibrated by using scaled radiation dose and film optical density. The gamma index passing rate was calculated with criteria set to a $3 \%$ dose difference and a $3 \mathrm{~mm}$ distance-to-agreement (DTA) for all dose points measured above a threshold of $10 \%$ of the global maximum dose.

Consistent with the liver SBRT protocol in our clinic, patient treatment setup started with verifying the isocenter which was initially placed in the target at simulation scan, then pre-treatment cone-beam CT on TrueBeam was registered to be matched with the volumetric dataset of average intensity projection (AVG) images imported from Philips Pinnacle Treatment Planning System. Image registration was performed automatically based on the region of interest, followed by manual refining performed by the treating physician to ensure the tumor was registered within the contoured ITV. The patient was re-positioned allowing 6 degrees of freedom (6-DOF) couch corrections according to the results of tumor soft tissue registration, and then the treatment delivery was initiated. All treatment processes of radiotherapy for SBRT, including patient set-up, tumor matching, and treatment delivery were monitored and asserted by the treating physician and physicist. ${ }^{28}$

\section{Dosimetric Comparison}

For each case, the two competing treatment plans (VMAT and WO-DCA) were compared in terms of initial treatment planning constraints and criteria of OARs [Dose maximum $\left(D_{\max }\right)$, for the spinal cord $(<18 \mathrm{~Gy})$, stomach ( $<28$ Gy), duodenum ( $<28$ Gy), small bowel ( $<28 \mathrm{~Gy})$, heart volume receiving $\mathrm{V}_{40 \mathrm{~Gy}}<10 \%$, mean doses $\left(\mathrm{D}_{\text {mean }}\right)$ of the liver $(<16 \mathrm{~Gy})$, dose $\mathrm{D}_{50 \%}$ of liver $(<15 \mathrm{~Gy})$ and normal liver volume (liver minus GTV volume) $\geq 700 \mathrm{cc}$ receiving $<15 \mathrm{~Gy}$ ]. The PTV coverage, including $\mathrm{D}_{98 \%}$ Gy as minimum dose, $\mathrm{D}_{2 \%}$ Gy as maximum dose, mean dose (Dmean), conformality index (CI) as recommended by ICRU 62, and Gradient Index (GI) as recommended by RTOG, were compared. The $\mathrm{CI}_{95}$ was calculated as the ratio between the volume enclosed by the $95 \%$ isodose volume and the part of the target receiving more than $95 \%$ $\left(\mathrm{CI}=\mathrm{V}_{\% 95} / \mathrm{TV} \% 95\right)$. The $95 \%$ isodose was chosen in reference to ICRU-62, to provide $95 \%$ target volume coverage. The GI was calculated, where $V_{R x}$ is the volume of the prescription isodose line, $\mathrm{V}_{50 \% \mathrm{Rx}}$ is the volume of the $50 \%$ of prescription isodose line, and $\mathrm{V}_{\mathrm{PTV}}$ is the volume of the PTV $\left(\mathrm{GI}=\mathrm{V}_{50 \% \mathrm{Rx} /} \mathrm{V}_{\mathrm{Rx}}\right){ }^{19,20}$ GI is used to evaluate 
Table 2 Plan Quality Parameters of VMAT and WO-DCA Plans

\begin{tabular}{|c|c|c|c|c|}
\hline Variable & WO-DCA & VMAT & p-value & t-value \\
\hline \multicolumn{5}{|l|}{ PTV } \\
\hline$D_{\text {mean }}(G y)$ & $54.65(51.30-60.23)$ & $53.76(51.90-60.73)$ & 0.90 & - \\
\hline$D_{\% 98}(G y)$ & $54.65(41.30-60.70)$ & $50.38(40.11-59.80)$ & 0.67 & - \\
\hline$D_{\% 2}(G y)$ & $58.00(53.98-64.69)$ & $57.22(57.7 I-68.59)$ & 0.72 & - \\
\hline \multicolumn{5}{|l|}{ Quality Parameters } \\
\hline BOT $(\min / f x)$ & $3.26(2.79-3.87)$ & $4.43(3.23-5.86)$ & $<0.001$ & - \\
\hline MU & 1860 (1709-2205) & 2705 (1977-3350) & $<0.001$ & - \\
\hline $\mathbf{C l}$ & I.I (I-I.I7) & I.I (I-I.09) & 0.96 & - \\
\hline GI & $4.0(3.21-8.2)$ & $4.0(3.7 I-8 . I)$ & 0.94 & 0.19 \\
\hline QA pass rate (\%) & $97.06 \pm 1.3$ & $93.00 \pm 1.66$ & $<0.001$ & 8.87 \\
\hline
\end{tabular}

Notes: p-value, Mann-Whitney $U$ test; t-value, Student's $t$-test results; statistically significant $\mathrm{p}$-values are highlighted in bold; mean \pm SD (range) and median (minimummaximum) values were reported.

Abbreviations: PTV, planning target volume; BOT, beam on time; Gy, Gray; $D_{\text {mean }}$, mean dose; $\mathrm{D}_{\% \mathrm{x}}$, dose on $\mathrm{X} \%$ volume; min/fx, minute of per fraction; MU, monitor units; $\mathrm{Cl}$, conformity index; $\mathrm{Gl}$, gradient index.

dose sparing in the healthy liver volume. The smaller GI values represent faster dose fall-off. The MUs per fraction and the beam on time (BOT) were also recorded during the phantom QA measurements to determine delivery time per fraction of each technique.

\section{Statistical Analysis}

The data were analyzed by using SPSS Statistics for Windows, Version 23.0 (IBM SPSS Statistics for Windows, IBMCorp Version 23.0. Armonk, NY). The data were assessed with the Shapiro-Wilk test in terms of deciding whether they were parametric. All parameters except $\mathrm{V}_{\text {liver }}-\mathrm{V}_{15 \mathrm{~Gy}} \geq 700 \mathrm{cc}$, $\mathrm{D}_{\max }<18$ Gy spinal cord, and QA pass rate were found to be nonparametric. The comparison of parametric data was performed by Student's $t$-test. The comparisons of nonparametric data were assessed using Mann-Whitney $U$ tests. The nonparametric data were given as medians, minimum and maximum values. The parametric data were expressed with mean \pm SD. A confidence interval of $95 \%$ and a two-tailed p-value of $\leq 0.05$ indicated statistical significance.

\section{Results}

\section{Plan Quality}

All PTV coverage, CI, GI, BOT (per fraction treatment time), and MU are detailed in Table 2. All the plans were clinically acceptable, as at least $95 \%$ of PTV receiving $95 \%$ of the prescribed dose, while the comparison of PTV minimum $\left(\mathrm{D}_{98 \%}\right)$, maximum $\left(\mathrm{D}_{2 \%}\right)$, and mean $\left(D_{\text {mean }}\right)$ dose parameters were not statistically significant. The mean PTV values were 54.65 (51.30-60.23) and
53.76 (51.90-60.73) in WO-DCA and VMAT techniques, respectively $(\mathrm{p}=0.9)$. The $\mathrm{D}_{98 \%}(\mathrm{p}=0.67)$ and the $\mathrm{D}_{2 \%}(\mathrm{p}=$ $0.72)$ values were similar between treatment techniques. The mean value of the QA pass rate to compare treatment was significantly higher with WO-DCA $(\mathrm{p}<0.001$ and $\mathrm{t}=$ 8.87). The treatment duration (BOT, $\mathrm{p}<0.001)$ and delivered radiation burden $(\mathrm{MU}, \mathrm{p}<0.001)$ were significantly lower with WO-DCA. The CI and GI were not statistically different between treatment techniques $(\mathrm{p}=0.96$ and $\mathrm{p}=$ 0.91). The axial, sagittal, and coronal views displaying dose distribution for VMAT and WO-DCA are shown in Figure 3 and plan quality metrics are displayed in Figure 4.

\section{OAR Doses}

The comparison of OAR DVH values is shown in Table 3. The dose distribution for the liver was assessed and compared in three groups: $\mathrm{V}_{\text {Liver }}-\mathrm{V}_{15 \mathrm{~Gy}} \geq 700 \mathrm{cc}, \mathrm{D}_{50 \%}<15$ $\mathrm{Gy}$, and $\mathrm{D}_{\text {mean }}<16 \mathrm{~Gy}$. The treatment techniques were not statistically different in these parameters. As such, there was no statistical difference in $\mathrm{D}_{\max }$ for the spinal cord, stomach, duodenum, small bowel values, and $\mathrm{V}_{40 \mathrm{~Gy}}$ for heart values.

\section{Discussion}

We have investigated whether WO-DCA liver SBRT might minimize small-field dosimetry errors without MLC interplay effects of respiratory motion as a result of elimination of MLC beam modulation through the target in liver SBRT for tumors away from critical structures. 


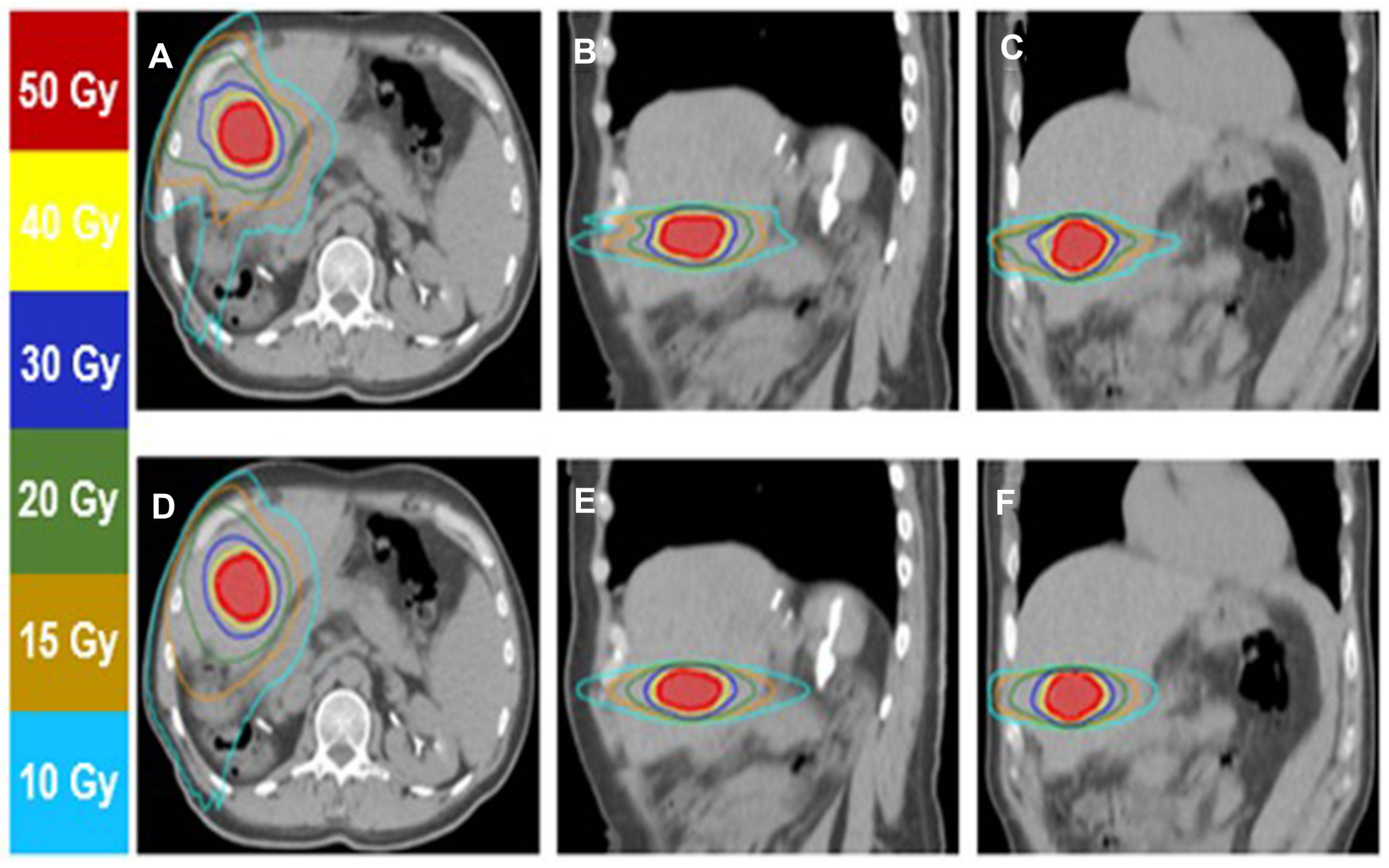

Figure 3 Comparison of a VMAT vs WO-DCA plan for the sample case described in the text: VMAT plan; (A) Axial (B) Sagittal, and (C) Coronal view, and WO-DCA plan; (D) Axial (E) Sagittal, and (F) Coronal view. The PTVs are defined by the red color.

Present results uncovered the WO-DCA technique's attainability to provide non-inferior OAR and target doses with roughly $45 \%$ lower radiation exposure and almost $33 \%$ faster treatment conveyance. Therefore, this technique may facilitate same-day SBRT with the potential elimination of patient-specific VMAT QA.

SBRT is fundamentally different from conventional radiotherapy with the unique ability to deliver a single or a few fractions (usually $\leq 5-8$ fractions) of massive dosages of ionizing radiation with high targeting exactness encompassing tumors via rapid dose fall-off gradients. Consequently, fostering the most efficient and viable SBRT treatment procedure has become a crucial issue for researchers. ${ }^{29,30}$ Dong et al compared $4 \pi$ plans with the 14 to 22 non-coplanar static beam IMRT plans among ten liver SBRT cases formerly managed with 50 to $60 \mathrm{~Gy}$ in 5 fractions utilizing VMAT, ${ }^{31}$ optimized to accomplish the objective of $95 \%$ of the PTV covered by $100 \%$ of the prescription dose, and concluded that the $4 \pi$ plans significantly and consistently yielded better target coverage and critical OAR protection. However, Dong et al did not report the total fraction time per patient by increasing the number of non-coplanar fields to provide the mentioned advantage. Resultantly, we believe that 22 non-coplanar fields per fraction to deliver SBRT would be clinically unpractical for currently existing LINAC clinical workflows. In contrast, our particular WO-DCA SBRT proposal could afford practically much faster and clinically more efficient SBRT delivery for selected liver metastases.

First described for lung SBRT protocols, which established the groundwork for liver SBRT, the fundamental intrafraction and interfraction respiratory motion of tumor and relevant solutions have been studied by many researchers. ${ }^{32-35}$ Small amplitude fluctuations of intrafraction and interfraction motion within $\pm 3 \mathrm{~mm}$ have been documented during a mean time of $35 \pm 7$ minutes from the setup localization of the tumor to the end-fraction CBCT. $^{34}$ Although there is no clear-cut consent and the minimum demanded CTV-to-PTV margin being a median of 5-mm (range: $3-7 \mathrm{~mm}$ ) in lung SBRT, ${ }^{36}$ a uniform 5-mm PTV margin around the ITV was proposed to be adequate to defeat these potential installation errors in liver SBRT. ${ }^{37,38}$ Aside from PTV margin, the interaction between MLC modulation and gantry rotation as 
A

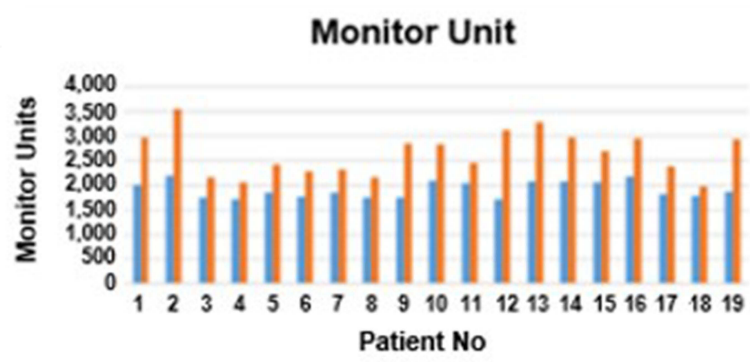

B

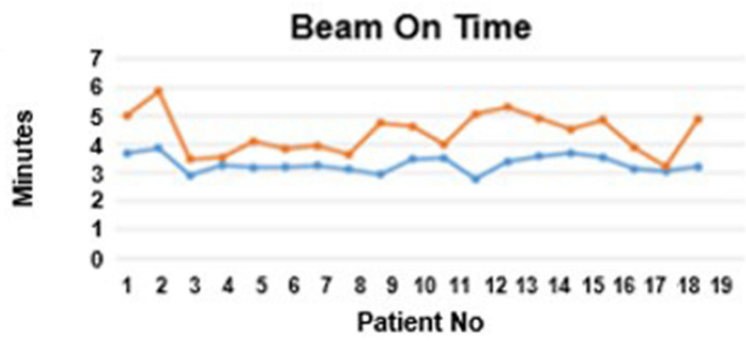

C

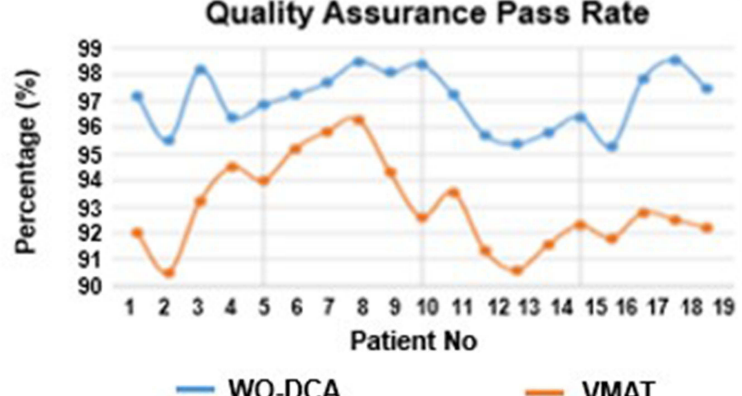

Figure 4 Treatment quality parameters having (A) monitor unit (MU); (B) beam on time (BOT); (C) quality assurance pass rate (QA pass rate) for VMAT vs WO-DCA plans. For VMAT vs WO-DCA plans; median values of MU were $2705 \mathrm{MU}$ and 1860 MU, median values of BOT were $4.43 \mathrm{~min}$ and $3.26 \mathrm{~min}$, and median values of $\mathrm{QA}$ pass rates were $93.00 \pm 1.66 \%$ and $97.06 \pm 1.3 \%$, respectively.

a function of tumor movement may provoke substantial dose turbidity in highly modulated VMAT plans in each fraction of liver SBRT. In our investigation, we have shown a $33 \%$ reduction in the mean irradiation delivery time per fraction for liver SBRT with the WO-DCA treatment technique as opposed to the standard VMAT procedure (4.40 vs.3.31 min for WO-DCA; $\mathrm{p}<0.0001$ ). Additionally, the plan stability was increased in this BOT due to better delivery accuracy of WO-DCA plans to eliminate the major concerns of small-field dosimetry errors and MLC interplay effects in contrast with the highly modulated VMAT plans.

The SBRT by VMAT has been a preferred technique over the conventional static field or IMRT techniques. ${ }^{39-41}$ Verbakel et al underscored VMAT to sanction delivery of hypofractionated doses in a much shorter time and more conformally than conventional SBRT with ten noncoplanar static fields for peripherally located stage I lung cancers. ${ }^{41}$ Nevertheless, there has been warning that VMAT-based SBRT could be susceptible and sensitive to dose inconsistencies based on the interplay effect of MLC sequence and tumor movement. ${ }^{42,43}$ Jiang et al documented the maximum variation around the mean dose of $>$ $30 \%$ for one-field IMRT, dilution up to $8 \%$ for all five fields in a single fraction, and $<1-2 \%$ within 30 fractions. They have additionally called attention to the lower dose rate to reduce the motion-related dose variations for the lung IMRT when no motion mitigation techniques are used. ${ }^{44}$ Court et al calculated the expected dose distribution by blurring the static dose distribution with the target motion and the dose error due to the interplay effect by comparing the delivered dose with the anticipated dose distribution and concluded that the interplay between the motions was increased with plan intricacy, target magnitude and period, while it averaged out after increasing the number of fractions. ${ }^{43}$ Moreover, Tyler et al likewise acknowledged that the SBRT delivery by VMAT was

Table 3 Average Dosimetric Results for Organs at Risk Sparing for VMAT and WO-DCA

\begin{tabular}{|c|c|c|c|c|}
\hline Variable & WO-DCA & VMAT & p-value & t-value \\
\hline Spinal cord $D_{\max }(G y)$ & $7.38 \pm 3.74$ & $7.35 \pm 2.57$ & 0.98 & 0.19 \\
\hline Stomach $D_{\max }(G y)$ & $4.38(0-19.69)$ & $4.09(0-17.72)$ & 0.99 & - \\
\hline Duodenum $D_{\max }(G y)$ & $0.49(0.10-12.08)$ & $0.59(0.10-13.04)$ & 0.90 & - \\
\hline Small Bowel $D_{\max }(G y)$ & $0.69(0.13-10.62)$ & $1.17(13.4-99.89)$ & 0.86 & - \\
\hline HeartV $_{40 G y}<10 \%(\%)$ & $0.11(0-6.09)$ & $0.11(0-6.39)$ & 0.90 & - \\
\hline$V_{\text {Liver }}-V_{15 G y} \geq 700 \mathrm{cc}$ & II $56.75 \pm 264.70$ & $1167.06 \pm 274.30$ & 0.90 & -0.11 \\
\hline Liver $D_{50 \%}<15$ Gy (Gy) & $1.45(0-12.17)$ & $1.84(0-11.85)$ & 0.76 & - \\
\hline Liver $D_{\text {mean }}(G y)$ & $5.56(1.96-13.43)$ & $5.68(2.02-12.00)$ & 0.94 & - \\
\hline
\end{tabular}

Notes: $\mathrm{p}$-value, Mann-Whitney $U$ test; t-value, Student's $t$-test results; statistically significant $\mathrm{p}$ - and t-values are highlighted in bold; mean \pm SD (range) and median (minimum-maximum) values were reported.

Abbreviations: Gy, Gray; \%, percent volume; $D_{\max }$, maximum dose; $D_{\text {mean }}$, mean dose; $D_{\% x}$, dose on $X \%$ volume; $V_{G y}$ volume receiving $X$ Gy dose. 
increasing the interplay effect with a maximum deviation of $4.8 \%$ in dose received by at least $1 \%$ (D1\%) of the target volume. ${ }^{45}$ Kubo et al analyzed and showed the significant impact of the number of breaths during irradiation to the dose variation for lung SBRT by VMAT, ${ }^{21}$ noting the importance of increasing number of breaths to decrease dose variability and outlined a suitability criteria of $>40$ breathes/two partial VMAT arcs (approximately 16 breaths per minute) to keep variability within $3 \%$. On the other hand, forward planning-based DCA delivery was shown to be efficient in lung SBRT by degrading the worries of the interplay effect. ${ }^{21-25}$ The overall goal with DCA was to reduce treatment time with lower geometric complexity, which may diminish the risk of interplay errors induced by respiratory motion. As Pokhrel et al previously emphasized, flattening filter free (FFF) VMAT for SBRT of lung lesions would improve dose coverage at tumor-lung interface compared to flattened beams, ${ }^{46}$ and additionally, there is growing evidence for FFF VMAT of various other tumor sites including the liver, brain, prostate, cervix cancers. ${ }^{47}$ Therefore, FFF VMAT could logically be compared, however, as we do not use FFF but 6 MV FF VMAT in our clinic, we hypothesized and tried to obtain a more efficient WO-DCA technique based on DCA treatment without MLC modulation in target to potentially minimize small-field dosimetry errors and MLC interplay effects. If we had used FFF for 6MV photon energy in our clinic and followed the identical plans for both treatment techniques in this investigation, the impact would most likely be the same on both arms, and the proportionate difference between the two planning techniques would most probably not have been altered.

To the best of our knowledge, small-field dosimetry error of MLC interplay effects of the respiratory motion in VMAT SBRT has not been studied in liver SBRT until now. Therefore, possible error risk is extrapolated due to the same basic principles of lung SBRT. Pokhrel et al have emphasized the possible adoption of DCA technique to other disease sites (including hypofractionated centrally located lung lesions, stereotactic treatment of brain or abdominal/pelvic lesions including liver SBRT) to ease the process. ${ }^{26}$ As we have already routinely conducted 4DCT and ITV approach for motion management for our liver targets, in this study, we compared installation of the DCA strategy consisting of 2 arcs and WO-DCA. Our present WODCA technique did not just enable a faster SBRT delivery compared to VMAT but additionally allowed more precise dose delivery in areas of tissue interfaces throughout the target volume with no MLC modulation.

In VMAT SBRT, gantry angles, dose rate, and the MLC positions vary during the radiation delivery, where the QA procedures needed to be able to detect all planning- and machine-related errors for the safe conveyance of the SBRT procedure. ${ }^{28,48}$ Liang et al analyzed three VMAT QA systems regarding their sensitivity to machine errors, including the gantry angles, MLC positions, and LINAC outputs. The authors proclaimed that neither of them was satisfactorily sensitive to the simulated output errors with their pros and cons related to sensitivity in different parameters. VMAT delivers the optimized dose distributions by numerous small beamlet-based intensity modulations using a combination of several separate MLC segments per arc. In contrast, the DCA conveys precise doses using dynamic MLCs and achieves required isodose distributions by utilizing only a couple of static beams. Based on this, WO-DCA could eliminate patient-specific VMAT QA and reduce the workload, which may enable same-day liver SBRT treatments.

We found no meaningful OAR dosimetric endpoint "disagreements" between the VMAT and novel WODCA techniques, ${ }^{49,50}$ as both passed the RTOG-0438 protocol assent criteria for clinically admissible SBRT. We need to call attention to the fact that the target selection in our study was the key to deliver a total of $50 \mathrm{~Gy}$ in 4 fractions by including only tumors at least $2-\mathrm{cm}$ away in all directions of any critical structures. Hence, we did not need the VMAT technique, which modulates the dose density, to spare a critical OAR compared to WO-DCA. Therefore, our WO-DCA technique has provided interchangeable dose distributions both in OARs and target lesions due to the initial compulsory selection of the tumor location and might be deemed sufficiently faster and more stable than VMAT in these tumors.

The findings of the present dosimetric study are novel for SBRT of metastatic liver tumors, and need to be confirmed by supplementary investigations. Our principal shortcoming was the selection of metastatic tumor localization to reduce the need for intensity modulation, and our cohort included only metastatic sites at least $2 \mathrm{~cm}$ away from organs at risk, revealing lesions cumulated in comparable liver segments. Therefore, corresponding results need cautious interpretation, and they should not be generalized to all liver metastases. The lack of post-treatment radiological scans following WO-DCA further restricted our long-term clinical understanding due to the dosimetric 
nature of our study. The variability in motion management strategy might present another handicap, where our routine involved 4D-CT, ITV approach, planning with cc convolution algorithm, and calculation on AVG data. Because the SBRT literature on motion management techniques was developed and evolved mainly in the lung, we could primarily extrapolate and explain our findings based on lung research. Overall, WO-DCA seems to be useful in decreasing the overall treatment time and overall treatment load in an overloaded radiation oncology facility, as well as the total radiation load delivered per patient. Since the nature of the target has no effect on the predicted delivery advantage, it might also be encouraged to explore WODCA in selected primary liver tumors meeting the eligibility criteria.

\section{Conclusion}

Present dosimetric results exhibited that liver SBRT plans with the novel WO-DCA technique could satisfactorily accomplish non-inferior delivery for selected lesions and enable up to $45 \%$ reduction in the cumulative quantity of MUs delivered due to less beam modulation across the ITV as opposed to VMAT plans. The WO-DCA technique ensured the significant one-third reduction in the BOT per fraction and theoretical duration of treatment slots, notwithstanding the possibility of bypassing patient-specific QA due to significantly reliable WO-DCA plans without significant intensity modulation. Therefore, our novel WODCA technique in liver SBRT of select metastatic tumors at least $2 \mathrm{~cm}$ away from the OARs seems to be a dependable and safe treatment approach in contrast to the standard ITV-dependent VMAT technique.

\section{Data Sharing Statement}

Data cannot be shared publicly because the data are owned and saved by Vehbi Koc Foundation Health Services. Data are available from the American Hospital Radiation Oncology Institutional Data Access/Ethics Committee (contact via Koc University Ethics Committee) for researchers who meet the criteria for access to confidential data: contact address, chr@ku.edu.tr.

\section{Author Contributions}

All authors made substantial contributions to the conception and design, acquisition of data, or analysis and interpretation of data; took part in drafting the article or revising it critically for important intellectual content; agreed to submit to the current journal; gave final approval of the version to be published; and agree to be accountable for all aspects of the work.

\section{Funding}

None to declare.

\section{Disclosure}

The authors have declared that no competing interests exist.

\section{References}

1. Kok END, Jansen EPM, Heeres BC, et al. High versus low dose stereotactic body radiation therapy for hepatic metastases. Clin Transl Radiat Oncol. 2020;20:45-50. doi:10.1016/j.ctro.2019.11.004

2. Nordlinger B, Sorbye H, Glimelius B, et al. Perioperative FOLFOX4 chemotherapy and surgery versus surgery alone for resectable liver metastases from colorectal cancer (EORTC 40983): long-term results of a randomised, controlled, phase 3 trial. Lancet Oncol. 2013;14 (12):1208-1215. doi:10.1016/S1470-2045(13)70447-9

3. Smith JJ, D'Angelica MI. Surgical management of hepatic metastases of colorectal cancer. Hematol Oncol Clin North Am. 2015;29 (1):61-84. doi:10.1016/j.hoc.2014.09.003

4. Hui TC, Kwan J, Pua U. Advanced techniques in the percutaneous ablation of liver tumours. Diagnostics. 2021;11(4). doi:10.3390/ diagnostics 11040585

5. Chalkidou A, Macmillan T, Grzeda MT, et al. Stereotactic ablative body radiotherapy in patients with oligometastatic cancers: a prospective, registry-based, single-arm, observational, evaluation study. Lancet Oncol. 2021;22(1):98-106. doi:10.1016/S14702045(20)30537-4

6. Katz AW, Carey-Sampson M, Muhs AG, Milano MT, Schell MC, Okunieff P. Hypofractionated stereotactic body radiation therapy (SBRT) for limited hepatic metastases. Int $J$ Radiat Oncol Biol Phys. 2007;67(3):793-798. doi:10.1016/j.ijrobp.2006.10.025

7. Rule W, Timmerman R, Tong L, et al. Phase I dose-escalation study of stereotactic body radiotherapy in patients with hepatic metastases. Ann Surg Oncol. 2011;18(4):1081-1087. doi:10.1245/s10434-010-1405-5

8. Rusthoven KE, Kavanagh BD, Cardenes H, et al. Multi-institutional phase I/II trial of stereotactic body radiation therapy for liver metastases. J Clin Oncol. 2009;27(10):1572-1578. doi:10.1200/ jco.2008.19.6329

9. McPartlin A, Swaminath A, Wang R, et al. Long-term outcomes of phase 1 and 2 studies of SBRT for hepatic colorectal metastases. Int J Radiat Oncol Biol Phys. 2017;99(2):388-395. doi:10.1016/j. ijrobp.2017.04.010

10. Scorsetti M, Comito T, Clerici E, et al. Phase II trial on SBRT for unresectable liver metastases: long-term outcome and prognostic factors of survival after 5 years of follow-up. Radiat Oncol. 2018;13(1):234. doi:10.1186/s13014-018-1185-9

11. Wang PM, Hsu WC, Chung NN, et al. Feasibility of stereotactic body radiation therapy with volumetric modulated arc therapy and high intensity photon beams for hepatocellular carcinoma patients. Radiat Oncol. 2014;9:18. doi:10.1186/1748-717X-9-18

12. Jung J, Yoon SM, Kim SY, et al. Radiation-induced liver disease after stereotactic body radiotherapy for small hepatocellular carcinoma: clinical and dose-volumetric parameters. Radiat Oncol. 2013;8:249. doi:10.1186/1748-717X-8-249

13. Long Y, Liang Y, Li S, et al. Therapeutic outcome and related predictors of stereotactic body radiotherapy for small liver-confined HCC: a systematic review and meta-analysis of observational studies. Radiat Oncol. 2021;16(1):68. doi:10.1186/s13014-021-01761-1 
14. Riou O, Llacer Moscardo C, Fenoglietto P, et al. SBRT planning for liver metastases: a focus on immobilization, motion management and planning imaging techniques. Rep Pract Oncol Radiother. 2017;22 (2):103-110. doi:10.1016/j.rpor.2017.02.006

15. Takeda A, Sanuki N, Tsurugai Y, Oku Y, Aoki Y. Stereotactic body radiotherapy for patients with oligometastases from colorectal cancer: risk-adapted dose prescription with a maximum dose of 83-100 Gy in five fractions. J Radiat Res. 2016;57(4):400-405. doi:10.1093/jrr/rrw029

16. Swaminath A, Massey C, Brierley JD, et al. Accumulated delivered dose response of stereotactic body radiation therapy for liver metastases. Int J Radiat Oncol Biol Phys. 2015;93(3):639-648. doi:10.1016/j.ijrobp.2015.07.2273

17. Van den Begin R, Engels B, Boussaer M, et al. Motion management during SBRT for oligometastatic cancer: results of a prospective phase II trial. Radiother Oncol. 2016;119(3):519-524. doi:10.1016/ j.radonc.2016.04.020

18. Van den Begin R, Engels B, Gevaert T, et al. Impact of inadequate respiratory motion management in SBRT for oligometastatic colorectal cancer. Radiother Oncol. 2014;113(2):235-239. doi:10.1016/j. radonc.2014.11.005

19. Lu L, Ouyang Z, Lin S, Mastroianni A, Stephans KL, Xia P. Dosimetric assessment of patient-specific breath-hold reproducibility on liver motion for SBRT planning. J Appl Clin Med Phys. 2020;21:77-83. doi:10.1002/acm2.12887

20. Stathakis S, Narayanasamy G, Licon AL, et al. A dosimetric comparison between volumetric-modulated arc therapy and dynamic conformal arc therapy in SBRT. Liver. 2019;82(55):5.

21. Kubo K, Monzen H, Tamura M, et al. Minimizing dose variation from the interplay effect in stereotactic radiation therapy using volumetric modulated arc therapy for lung cancer. J Appl Clin Med Phys. 2018;19(2):121-127. doi:10.1002/acm2.12264

22. Ross CC, Kim JJ, Chen ZJ, Grew DJ, Chang BW, Decker RH. A novel modified dynamic conformal arc technique for treatment of peripheral lung tumors using stereotactic body radiation therapy. Pract Radiat Oncol. 2011;1(2):126-134. doi:10.1016/j. prro.2010.11.002

23. Bokrantz R, Wedenberg M, Sandwall P. Dynamic conformal arcs for lung stereotactic body radiation therapy: a comparison with volumetric-modulated arc therapy. J Appl Clin Med Phys. 2019;21. doi:10.1002/acm2.12800

24. Pokhrel D, Halfman M, Sanford L. A simple, yet novel hybrid-dynamic conformal arc therapy planning via flattening filter-free beam for lung stereotactic body radiotherapy. $J$ Appl Clin Med Phys. 2020;21(6):83-92. doi:10.1002/acm2.12868

25. Pokhrel D, Halfman M, Sanford L, Chen Q, Kudrimoti M. A novel, yet simple MLC-based 3D-crossfire technique for spatially fractionated GRID therapy treatment of deep-seated bulky tumors. $J$ Appl Clin Med Phys. 2020;21(3):68-74. doi:10.1002/acm2.12826

26. Pokhrel D, Visak J, Sanford L. A novel and clinically useful dynamic conformal arc (DCA)-based VMAT planning technique for lung SBRT. J Appl Clin Med Phys. 2020;21(7):29-38. doi:10.1002/acm2.12878

27. Katz A, Winter K, Dawson L, et al. RTOG 0438: a phase I trial of highly conformal radiation therapy for patients with liver metastases. J Clin Oncol. 2012;30:257. doi:10.1200/jco.2012.30.4_suppl.257

28. Saglam Y, Bolukbasi Y, Atasoy A, et al. Quality assurance in stereotactic radiosurgery and stereotactic body radiotherapy. Adv Res. 2020:22-33. doi:10.9734/air/2020/v21i730216

29. Kim S, Palta J. The physics of stereotactic radiosurgery. In: Chin LS, Regine WF, editors. Principles and Practice of Stereotactic Radiosurgery. New York: Springer; 2008:33-50.

30. Saw C, Bao S, Li S. A review on the technical and dosimetric aspects of stereotactic body radiation therapy (SBRT). J Radiat Oncol. 2012;1:317-322. doi:10.1007/s13566-012-0025-z

31. Dong P, Lee P, Ruan D, et al. $4 \pi$ non-coplanar liver SBRT: a novel delivery technique. Int $J$ Radiat Oncol Biol Phys. 2013;85 (5):1360-1366. doi:10.1016/j.ijrobp.2012.09.028
32. Hoogeman MS, Nuyttens JJ, Levendag PC, Heijmen BJ. Time dependence of intrafraction patient motion assessed by repeat stereoscopic imaging. Int $J$ Radiat Oncol Biol Phys. 2008;70(2):609-618. doi:10.1016/j.ijrobp.2007.08.066

33. Purdie TG, Bissonnette JP, Franks K, et al. Cone-beam computed tomography for on-line image guidance of lung stereotactic radiotherapy: localization, verification, and intrafraction tumor position. Int J Radiat Oncol Biol Phys. 2007;68(1):243-252. doi:10.1016/j. ijrobp.2006.12.022

34. Bissonnette JP, Franks KN, Purdie TG, et al. Quantifying interfraction and intrafraction tumor motion in lung stereotactic body radiotherapy using respiration-correlated cone beam computed tomography. Int J Radiat Oncol Biol Phys. 2009;75(3):688-695. doi:10.1016/j.ijrobp.2008.11.066

35. Li W, Purdie TG, Taremi M, et al. Effect of immobilization and performance status on intrafraction motion for stereotactic lung radiotherapy: analysis of 133 patients. Int J Radiat Oncol Biol Phys. 2011;81(5):1568-1575. doi:10.1016/j.ijrobp.2010.09.035

36. Guckenberger M, Andratschke N, Dieckmann K, et al. ESTRO ACROP consensus guideline on implementation and practice of stereotactic body radiotherapy for peripherally located early stage non-small cell lung cancer. Radiother Oncol. 2017;124(1):11-17. doi:10.1016/j.radonc.2017.05.012

37. Gargett M, Haddad C, Kneebone A, Booth JT, Hardcastle N. Clinical impact of removing respiratory motion during liver SABR. Radiat Oncol. 2019;14(1):93. doi:10.1186/s13014-019-1300-6

38. Liu M, Cygler JE, Vandervoort E. Patient-specific PTV margins for liver stereotactic body radiation therapy determined using support vector classification with an early warning system for margin adaptation. Med Phys. 2020;47(10):5172-5182. doi:10.1002/ mp.14419

39. Bignardi M, Cozzi L, Fogliata A, et al. Critical appraisal of volumetric modulated arc therapy in stereotactic body radiation therapy for metastases to abdominal lymph nodes. Int J Radiat Oncol Biol Phys. 2009;75:1570-1577. doi:10.1016/j.ijrobp.2009.05.035

40. Malouff TD, Seneviratne D, Stross WC, et al. Public interest in stereotactic body radiation therapy (SBRT) and stereotactic radiosurgery (SRS) in the United States. J Radiosurg SBRT. 2020;6 (4):311-315.

41. Verbakel WF, Senan S, Cuijpers JP, Slotman BJ, Lagerwaard FJ. Rapid delivery of stereotactic radiotherapy for peripheral lung tumors using volumetric intensity-modulated arcs. Radiother Oncol. 2009;93 (1):122-124. doi:10.1016/j.radonc.2009.05.020

42. Bortfeld T, Jokivarsi K, Goitein M, Kung J, Jiang SB. Effects of intra-fraction motion on IMRT dose delivery: statistical analysis and simulation. Phys Med Biol. 2002;47(13):2203-2220. doi:10.1088/ 0031-9155/47/13/302

43. Court L, Wagar M, Berbeco R, et al. Evaluation of the interplay effect when using RapidArc to treat targets moving in the craniocaudal or right-left direction. Med Phys. 2010;37(1):4-11. doi:10.1118/ 1.3263614

44. Jiang SB, Pope C, Al Jarrah KM, Kung JH, Bortfeld T, Chen GT. An experimental investigation on intra-fractional organ motion effects in lung IMRT treatments. Phys Med Biol. 2003;48(12):1773-1784. doi:10.1088/0031-9155/48/12/307

45. Tyler MK. Quantification of interplay and gradient effects for lung stereotactic ablative radiotherapy (SABR) treatments. J Appl Clin Med Phys. 2016;17(1):158-166. doi:10.1120/jacmp.v17i1.5781

46. Pokhrel D, Halfman M, Sanford L. FFF-VMAT for SBRT of lung lesions: improves dose coverage at tumor-lung interface compared to flattened beams. J Appl Clin Med Phys. 2020;21(1):26-35. doi:10.1002/acm2.12764

47. Ghemis DM, Marcu LG. Progress and prospects of flattening filter free beam technology in radiosurgery and stereotactic body radiotherapy. Crit Rev Oncol Hematol. 2021;163:103396. doi:10.1016/j.critrevonc.2021.103396 
48. Liang B, Liu B, Zhou F, Yin FF, Wu Q. Comparisons of volumetric modulated arc therapy (VMAT) quality assurance (QA) systems: sensitivity analysis to machine errors. Radiat Oncol. 2016;11. doi:10.1186/s13014-016-0725-4

49. Michel R, Françoise I, Laure P, Anouchka M, Guillaume P, Sylvain K. Dose to organ at risk and dose prescription in liver SBRT. Rep Pract Oncol Radiother. 2017;22:96-102. doi:10.1016/j. rpor.2017.03.001
50. Pan CC, Kavanagh BD, Dawson LA, et al. Radiation-associated liver injury. Int J Radiat Oncol Biol Phys. 2010;76(3 Suppl):S94-100. doi:10.1016/j.ijrobp.2009.06.092

\section{Publish your work in this journal}

Therapeutics and Clinical Risk Management is an international, peerreviewed journal of clinical therapeutics and risk management, focusing on concise rapid reporting of clinical studies in all therapeutic areas, outcomes, safety, and programs for the effective, safe, and sustained use of medicines. This journal is indexed on PubMed Central, CAS,
EMBase, Scopus and the Elsevier Bibliographic databases. The manuscript management system is completely online and includes a very quick and fair peer-review system, which is all easy to use. Visit http://www.dovepress.com/testimonials.php to read real quotes from published authors. 